then placed in isolation until their own songs were analysed and compared with the tutor song and a novel song. On average, there was significant song copying. The birds were then exposed to the tutor song, or they were not exposed to a song (controls), in darkness, for $30 \mathrm{~min}$. One hour later the animals were perfused and their brains were prepared for immunocytochemical analysis of IEG protein product expression. The number of immunopositive cell nuclei in a standard size frame in the CHV, NCM and the lobus parolfactorius (LPO) was determined in a blind procedure. There was egr-1-like (ZENK) and Fos-like immunoreactivity in several forebrain regions, but not, or very little, in the conventional song control nuclei HVC, RA, Area X and IMAN, and in the LPO. Exposure to the tutor song led to a significant increase in the expression of both Fos $(t(11)=3.87 \mathrm{P}<0.01)$ and $\operatorname{ZENK}(\mathrm{t}(11)=2.72, \mathrm{P}<0.02)$ in the NCM, but not in the CHV, compared to controls. Furthermore, the strength of the IEG response in the NCM correlated significantly and positively with the number of song elements that the birds had copied from the tutor song (Fos: $\mathrm{r}=0.922, \mathrm{P}<0.01$; ZENK: $\mathrm{r}=0.829 \mathrm{P}<0.05$, respectively). This is the first time that song-induced IEG expression was found in song birds with a known history of song learning. Furthermore, for the first time localised immediate early gene expression has been found in response to tutor song exposure, that correlates with the strength of song learning.

\title{
Time and Space
}

TS1

DISCRIMINATION OF BIOLOGICAL MOTION (JOHANSSON'S DISPLAYS) BY NEWBORN CHICKS IMPRINTED ONTO POINT-LIGHT ANIMATION SEQUENCES

L. Regolin ${ }^{\prime *}$, L. Tommasi $^{l}$, and G. Vallortigara ${ }^{2}$

${ }^{l}$ Dipartimento di Psicologia Generale, Università degli Studi di Padova, Via Venezia 8, 35131 Padova, Italy and ${ }^{2}$ Dipartimento di Psicologia, Università degli Studi di Trieste, Via dell'Università 7, 34123 Trieste, Italy

Newborn chicks were isolated and exposed for two hours to point-light animation sequences depicting either a walking hen or a rotating cylinder. In a subsequent free-choice test chicks preferentially approached the novel stimulus, no matter of this being the walking hen or the rotating cylinder. In order to obtain equivalent local motion vectors, in a new experiment, 1-day and 2-day-old chicks were exposed either to a point-light animation sequence depicting a walking hen or to a "positionally scrambled" walking hen (i.e an animation sequence constituted of the same set of dots in motion as that employed for the walking hen, but with spatially randomised starting positions). Chicks of both ages proved able to discriminate the two animation sequences: males approaching preferentially the novel stimulus, and females the familiar one. These results indicate that discrimination was not based on local motion vectors, but rather on the temporally integrated motion sequence. 
TS2

\title{
PROCESSING SUBJECT-OBJECT DISTANCE IN A VISUAL RECOGNITION MEMORY SYSTEM
}

\author{
A.U. Nicol ${ }^{*}$, M.W. Brown ${ }^{2}$, and G. Horn ${ }^{l}$ \\ ${ }^{\prime}$ Sub-department of Animal Behaviour, Cambridge University, Cambridge CB3 8AA, and ${ }^{2}$ Anatomy \\ Department, Bristol University, Bristol BS8 1TD, UK
}

Visually naive domestic chicks if trained by extended exposure $(\sim 2 \mathrm{~h})$ to an object subsequently express a social attachment to that object (1) by approaching it in preference to an alternative stimulus; if the training stimulus recedes, the chick follows it. This behaviour implies that the chick learns the features of an object when exposed to it ('imprinting'), commits that information to memory, and has a mechanism for encoding subject-object distance $(d)$. Past studies have implicated a region of the forebrain, the intermediate and medial part of the hyperstriatum ventrale (IMHV), as a memory store for imprinting (2). Electrophysiological studies in behaving chicks have shown that the proportion of IMHV neurons responsive to a particular visual stimulus is higher in chicks trained with that stimulus than in chicks trained with another stimulus or in untrained chicks. Among the responsive neurons some respond selectively to the training stimulus and some encode a particular feature of that stimulus (its colour or shape). Do IMHV neurons also encode $d$ ? Chicks ( $24 \mathrm{~h}$ post hatch) were placed in running wheels and trained for $2 \mathrm{~h}$ by exposure to a rotating stimulus (a red box or blue cylinder). After training, each chick was prepared under anaesthesia for recording from IMHV neurons. The following day when the chick had recovered from anaesthesia it was returned to the running wheel. Action potentials from individual neurons were software sorted from multiunit activity and the neurons were tested for responsiveness to the training stimulus and the alternative stimulus at each of three distances $(d=0.5 \mathrm{~m}, 1 \mathrm{~m}, 2 \mathrm{~m})$. When tested with the training stimulus (3), certain neurons were $d$-sensitive, their responses varying significantly $(A N O V A, P<0.05)$ with $d$. Other, $d$-invariant neurons responded to the training stimulus irrespective of $d$. Subsequent studies (4) demonstrated that IMHV neurons may exhibit $d$-sensitivity or $d$ invariance not only for the training stimulus but also for the alternative stimulus. Thus neurons in the IMHV encode $d$ irrespective of the chick's imprinting experience. This and other behavioural studies (5) indicate that a neural mechanism for processing $d$ exists in chicks which have had little or no experience of variations in distance.

Supported by BBSRC. Thanks to Dr. Scarlett Pinnock.

Bolhuis (1991) Biol. Rev., 41:177; (2) Horn (1998) Trends Neurosci., 21:300; (3) Nicol et al. (1998) Eur. J. Neurosci., 10:34 (4) Nicol et al., (1999) Neuroreport, 10 (in press); (5) Nicol, (1999) this meeting. 
TS3

\section{INVARIANT VISUAL RECOGNITION IN A NAIVE BRAIN}

A.U. Nicol ${ }^{\prime}$, V.C. Mills, M.W. Brown ${ }^{2}$, and G.Horn ${ }^{l}$

${ }^{\prime}$ Sub-department of Animal Behaviour, Cambridge University, Cambridge CB3 8AA, and ${ }^{2}$ Anatomy Department, Bristol University, Bristol BS8 1TD, UK

Visually naive domestic chicks if exposed to an object (e.g. the mother hen or an artificial surrogate) for $\sim 2 \mathrm{~h}$ form a social attachment to it (1). Subsequently chicks approach the familiar stimulus in preference to other stimuli, and if that stimulus recedes the chicks follow it. This behaviour implies that chicks learn the characteristics of an object when exposed to it ('imprinting'), commit that information to memory, and have a mechanism for encoding the distance $(d)$ to the object. A long-held theory is that "the judgement we make of the distance of an object... is entirely the result of experience" (George Berkeley, 1709, A New Theory of Vision). However, chicks which have had minimal experience of variations in $d$ appear to modify their behaviour appropriately when $d$ varies (2): if $d$ increases they approach the object with greater vigour and if it decreases they approach less. Do chicks indeed perform $d$-invariant visual discriminations with no experience of exploration in extended space? Chicks were raised in a dark incubator until $\sim 24 \mathrm{~h}$ post hatch, then placed in running wheels and trained for $2 \mathrm{~h}$ by exposure to a rotating stimulus (a red box or blue cylinder). During training the stimulus was presented at $d=0.5 \mathrm{~m}$. After training, two preference tests were conducted: $1 \mathrm{~h}$ (test 1) and 24h (test 2) after completing training. In the tests serial 4 minute presentations were made of the training stimulus (twice) and the alternative stimulus (twice) in balanced order. A preference score was calculated expressing the chick's approach (wheel rotations) towards the training stimulus as a percentage of the total approach to both stimuli. Test 1 was conducted with the stimuli presented at either $d=0.5 \mathrm{~m}$ or $d=2 \mathrm{~m}$. In test 2 the stimuli were presented at the other distance. Although the mean preference score when the stimuli were presented at $d=2 \mathrm{~m}(68.1$ $\pm 2.4 \%)$ was lower $(P<0.01)$ than when $d=0.5 \mathrm{~m}(77.6 \pm 3.2 \%)$, the scores in both tests were above chance at $50 \%(P<0.001)$. These scores were influenced by neither the stimulus used in training nor the order in which $d$ was varied between tests. Thus chicks with no experience of interacting with an object in extended space can nonetheless recognise it when it is presented at an unfamiliar subject-object distance. This and other studies $(2,3)$ suggest that a neural mechanism for encoding subject-object distance exists with little or no experience of variations in this dimension.

Supported by BBSRC.

(1) Bolhuis (1991) Biol. Rev., 41:77; (2) Nicol et al., (1998) Eur. J. Neurosci., 10:34; (3) Nicol et al., (1999) this Meeting. 
TS4

PREFRONTAL CORTEX LESION OF RATS DISRUPT TIME ESTIMATION IN A REACTION TIME TASK

C. Risterucci, D. Terramorsi, and M. Amalric*

Functional and Cellular Neurobiology Laboratory, CNRS UPR 9013, Marseille, France

The medial prefrontal cortex (PFC) is known to be involved in a variety of cognitive processes including learning and memory, emotion, motivation and attention. Its potential role in motor function is however poorly understood, although clinical evidence has shown motor disturbance in patients with frontal lobe dysfunction. The effects of PFC lesion were thus tested in rats trained to perform a reaction time (RT) task. Animals were trained to hold a lever down until the onset of a visual stimulus and to release it rapidly afterwards (with a time limit of $600 \mathrm{~ms}$ ). A high attentional load was required to correctly perform the task as the visual cue was unpredictible and could occur after four variable and randomly generated intervals $(0.5,0.75,1$ and $1.25 \mathrm{~s})$. Furthermore, the time constraint to respond after the cue required adequate motor readiness. Local infusion of ibotenic acid into the medial PFC impaired RT performance by dramatically increasing premature responding (anticipatory response before the visual cue) and shortening RTs. The peaks of premature responses $50-100 \mathrm{~ms}$ prior to the various intervals preceding the cue disappeared after the lesion, suggesting a disruption of time estimation. These behavioral deficits were entirely abolished by the dopaminergic nerve terminals lesion in the nucleus accumbens whereas RT performance was not modified by the dopamine lesion alone. The recovery of preoperative performance revealed a reactivity of the dopaminergic mesolimbic system after a PFC lesion which may account for the speeding of RTs and the perturbation of time estimation. The behavioral inhibitory control of the PFC may thus play a critical role in the development of the psychotic symptoms related to the disruption of time estimation and attentional processes observed in schizophrenic patients.

\section{TS5}

DISCRIMINATION OF SPATIAL NOVELTY IN LABORATORY MICE: AN ONTOGENETIC ANALYSIS

C. Colozza*, L. Ricceri, and G. Calamandrei

Section of Comparative Psychology, Laboratory of Pathophysiology, Istituto Superiore di Sanità, Viale Regina Elena 299, 00161 Rome, Italy

The ability to recognise and remember the spatial characteristics of the surrounding environment is essential for surviving in a natural habitat. Most of experimental work on spatial discrimination has been carried out in adult rodents, using either terrestrial or water mazes. The present ontogenetic study investigated the emergence of spatial discrimination in CD-1 mice, using a "spatial" open-field test with four objects, namely a non-associative task in which responses to both new spatial rearrangement of familiar objects as well as to exposure to novel unfamiliar objects are measured ( Poucet, 1989; Thinus- 
Blanc et al., 1996; Usiello et al., 1998). Male and female mice were tested on postnatal day (pnd) 18, 28, 46 and 90 in a black square open-field arena, surrounded by a visually uniform environment. Testing consisted of seven successive 4 min sessions. During session 1 each mouse was individually placed into the empty arena and baseline levels of locomotory activity and general behaviour were recorded. From session 2 to 4 , four different plastic objects were present in the arena in a fixed configuration, but in session 5, the exposition of two of the four objects was changed. In session 6 the spatial configuration of the objects was the same as session 5, while in session 7 one of the familiar non-displaced object was substituted with a novel one. The time spent exploring the objects as well as general behaviour were recorded throughout the seven sessions. We found that pre-weaning mice (pnd 18) were unable to detect both object rearrangement and object novelty, suggesting immaturity in the process of recognising or remembering spatial information. On pnd 28 and 46 mice still did not react to object displacement, but they showed an adult-like response to object novelty, actively exploring the unfamiliar object placed in the arena. A clear response to object displacement emerged only at adulthood. No sex differences in this ontogenetic profile were evidenced. These findings show that the ability to discriminate subtle changes in the environment does not mature until adulthood in the mouse brain, whereas the reaction to a novel object is already present in periadolescent mice (pnd 28). It appears that the spatial open-field task, exploiting the natural propensity to explore the environment by rodents, may reveal more subtle deficits in spatial cognition than those evidenced by the use of either terrestrial or water mazes.

TS6

NAVIGATION BY PATH INTEGRATION AND VISUAL CUES IN RODENTS

A.S. Etienne*, R. Maurer, and A. Levy

Laboratoire d'Ethologie, FPSE, Université de Genève, Switzerland

Behavioural experiments and unit recordings in the hippocampus and related brain areas show that rodents navigate by using predominantly two complementary categories of spatial information : Path integration ( dead reckoning ) - probably a basic mechanism in all sedentary species - allows the animals to automatically update their current position in a familiar or new environment through self-motion cues. Stable landmark-place associations enable the subjects to find their way through familiar space and to pinpoint specific locations on a long-term basis. In total darkness, the golden Hamster (Mesocricetus auratus $W$.) hoards food through path integration. Throughout a hoarding excursion, the animal computes its current position with respect to the starting point at the nest on the basis of signals from locomotion. The animal can therefore return home along a direct path at any moment of its excursion. Furthermore, through the principle of vector addition, self-generated vector information can control the complete round trip leading from the nest to a familiar feeding place and back again to the nest. However, path integration based on purely internal references is open to rapidly accumulating errors. By itself, it can be used over limited excursions only. When possible, Hamsters and other rodents mainly rely on visual landmarks. In conflict situations between learned visual cues and vector information the animals often 
home along compromise directions, giving a greater weight either to vision or to path integration depending on the degree of conflict and/or the structure of the experimental space. The versatility of their behaviour suggests that they evaluate how much they can trust a given category of information. To optimise navigation, path integration and learned external references must cooperate. In Hamsters, episodic fixes on the familiar environment can reset a drifting path integrator and confirm a trajectory that is planned and executed through path integration. Path integration, on the other hand, helps the animal to use the right landmark at the right moment and to identify ambiguous external references. These behaviours match data from single unit recordings. Place and head direction cells are mainly driven by vision. In darkness, the cells' firing pattern first remains correlated with the external world thanks to motion cues, and then starts drifting. Resetting occurs if the light is turned on again. In conflict situations, place and head direction cells seem to depend on visual and/or motion cues according to their reliability. This correspondence between behaviour and single cell activity testifies to the high degree of spatial information processing by neurones of the hippocampus and functionally related brain areas.

\title{
TS7
}

\section{RHESUS MONKEYS USE GEOMETRIC AND NON GEOMETRIC SPATIAL INFORMATION TO ORIENT}

\author{
S. Gouteux, J. Vauclair, and C. Thinus-Blanc*. \\ Center for Research in Cognitive Neuroscience, CNRS, Marseille, France
}

The geometry of surfaces and a variety of local cues are constituent elements of spatial representations. Several studies have demonstrated in rats (e.g. Cheng, 1986) and toddlers (Hermer and Spelke, 1994) that geometric information predominates over local cues that are neglected even though they provide relevant information for solving the task. In contrast, human adults make a joint use of geometric and local information. The present series of experiments was aimed at investigating the use of the two categories of information in a spatial orientation task. The experimental apparatus was a rectangular room. Four identical boxes were located at the corners of the room. The monkey could see the experimenter hidding a reward in one of the boxes. After desorientation (slow rotations without vision), the animal was released and could freely search for the reward box. In Exp. 1,monkeys' ability to use geometry to reorient was tested. Exp. 2 was aimed at determining whether monkeys can make a joint use of geometric and non-geometric information. The small side of the room was entirely covered with a large blue panel either closely associated to (Exp. 3) or spatially dissociated (Exp. 4) from the reward box. Four different small patterns were located in the corners of the room in Exp. 4 and 5. Two of them were removed (Exp. 4) or all the four were left (Exp. 5) during the test. Finally, Exp. 6 and 7 investigated the effects of the size of the pattern on re-orientation. Data show that rhesus monkeys use the geometry of the room in the lack of any other information (Exp. 1), searching as often in the baited box than in its symetrically opposite. When a coloured wall was affixed to cne of the walls, either close to (Exp. 2) or dissociated (Exp.3) from the reward box, monkeys use it to make the distinction between the two symetrical corners. 
In contrast, small patterns in the corners (Exp. 4 and 5) are ignored. This « neglect » appears to be related to the small size of the patterns (Exp. 6 and 7). In summary, these data demonstrate that unlike toddlers, monkeys can make a joint use of geometric and non-geometric information. However, non-geometric cues need to be conspicuous enough so as to induce monkeys to use them. These data are discussed in relation with the notion of a possible geometric module and with the flexibility of spatial representations in the course of evolution.

(1) Cheng (1986) Cognition, 23:149; (2) Hermer \& Spelke (1994) Nature 370:57.

\section{TS8}

\section{LANDMARK-GUIDED REORIENTATION BY CHILDREN IN AN OPEN SPACE}

S. Gouteux ${ }^{1 *}$ and E. Spelke ${ }^{2}$

${ }^{l}$ Center for Research in Cognitive Neurosciense, CNRS, Marseille, France and ${ }^{2}$ Infant Cognition Laboratory, Department of Brain and Cognitive Sciences, MIT, Boston, USA

In a series of eight behavioral studies, we show that children do not use the spatial arrangement of objects to reorient themselves in a room that provides no geometric cues. All the experiments were designed in a circular open space, similar in important ways to spaces used in studies of animal spatial orientation. Boxes were arranged in the room such that their geometric arrangement specified the location of the hidden object. After a brief familiarization, the subject was mildly disoriented and then given the task of locating the hidden toy that he/she had previously hidden into one of the boxes. The first 6 studies examined what kinds of geometric and non-geometric information children at the age of 3 and 4 years utilize to reorient themselves. Results of the the first study showed that young children do not appear to use geometric cues provided by objects in the room (identical boxes arranged in a right triangle) when the room itself is symmetrical; children were random in their searching following disorientation. In the second study, distinctively shaped and colored boxes were arranged in the same pattern, and in this condition children were able to find the hidden object quite acurately. In the third and fourth studies, more salient geometric information was provided by arranging the identical boxes such that two of them were very close together or by placing tape on the floor marking the geometric shape defined by the boxes. In these conditions, children still did not appear to use the geometric cues to reorient themselves, performing at chance in finding the hidden toy. In the two remaining studies, children were provided with a large landmark cue (a red curtain on the wall of the room), and with a rectangular arrangement of boxes. This geometric information did not assist in children's reorientation. Taken together with the findings of previous experiments $(1,2)$, these results show that children are able to reorient with geometric information provided by the room itself (for example a rectangular room) but not with geometric information provided by an arrangement of separately movable object. Our results suggest that reorientation mechanism used by children is not a pure "geometric module" (3) that computes angles and distances relationships among all detectable landmarks. Instead, children's reorientation depends specifically on a representation of the shape of the extended surfaces layout. 
(1) Hermer \& Spelke (1994) Nature, 370, 57-59; (2) Hermer \& Spelke (1996) Cognition, 61:195; (3) Cheng, 1986, Cognition, 23:149

\section{TS9}

DEVELOPMENTAL ASPECTS OF MAXIMUM FINGER TAPPING TEMPO

J. Kowalska ${ }^{\prime}$, L. Do ${ }^{l}$, E. Szelag ${ }^{l}, K$. Rymarczyk ${ }^{l}$, and E. Pöppel ${ }^{2}$

'Department of Neurophysiology, Nencki Institute of Experimental Biology, 02-093 Warsaw, Poland and

${ }^{2}$ Institute of Medical Psychology, D-80336 Munich, Germany

Neuropsychological studies on temporal information processing suggest temporal constraints on at least two different ranges, namely a high-frequency level of approx. 30-40 ms and a more complex lowfrequency level of approx. 2-3 s. Between these two temporal domains, the level of voluntary motor control is located with a range of some hundreds ms. Accordingly, the aim of the present study was to test alterations in temporal constraints of motor control during child cognitive development in production of simple repetitive movements, such as the maximum finger tapping (MFT) task. We also studied associations of two, different components of tapping temporal structuring: the key-touching-time (KTT) and the inter-tap-interval (ITI). The former represents the end of an agonistic and the beginning of an antagonistic finger movements. Such a complex ballistic movement is highly automatized and reflects the execution of an action. The later constitutes an interval between two consecutive taps and reflects the planning and preparation to the movement. Sixty elementary school pupils classified into three age groups: 6-7-year-olds, 9-10-year-olds, 13-14-year-olds were asked to tap with their index finger on a button as fast as possible by the right and the left hand. The sequences of 50 consecutive taps were analysed. The results showed that the MFT tempo significantly increased with normal child development. Moreover, independently of the subjects' age, we found that: first, the acceleration in MFT tempo for the right (dominant) hand in comparison with the performance of the left hand, second, significantly longer KTT than ITI. This relationship was more distinct in boys than in girls. To sum up these observations, we conclude that although the MFT tempo increases with normal child development, the modifications in central timing do not influence the relationship between two tapping components. The lack of such consistent differences may indicate that both the execution and planning of movement undergo similar remodelling during ontogenesis. 
TS10

THE ANALYSIS OF THE TIME STRUCTURE OF BEHAVIOUR

M. Puopolo*, A. Venerosi, D. Santucci, E. Alleva, and F. Chiarotti

Sections of Comparative Psychology and Behavioural Pathophysiology, Lab. F.O.S, Istituto Superiore di Sanità, Roma, Italy

Frequencies and/or durations of behavioural items are usually analysed to assess the effects of the exposure to noxious agents. Moreover, the sequences of events and the time points at which they occur (transitions between behavioural items) are frequently recorded. Nevertheless, the information about the temporal structure of behaviour is often neglected and many results are missed, probably due to the lack of an easy-to-use method. The time structure of behaviour can be described by the transition rates between behavioural items, which represent the chance per time unit that an item is followed by the other one, and the termination rates of each item, which represent the chance per time unit that the item is interrupted (1). Data on the sequences of events and the time points at which they occur can be considered failure time data. Therefore, the Cox proportional hazards model can be used to assess alterations of transition (termination) rates caused by experimental treatments (2). Data on the effects of prenatal exposure to different doses of pharmacological agent on aggressive interactions in adult male mice, and on the effects of neonatal PCB exposure on social interactions in periadolescent mice are used to show the application of the method. In the former experiment, adult male mice prenatally exposed to low or high concentrations of a toxicant, underwent aggressive interactions in 20-min agonistic encounters with a standard opponent. In the experiment on effects of PCB exposure, male littermates were randomly assigned to 5 treatment groups: control, low and high doses of two different PCBs. Mice underwent social encounters with an animal of the same sex, age and treatment. In both the experiments, the encounters were videotaped and the behaviour was subsequently scored. In conclusion, our results show that the alterations of the time structure of behaviour caused by pharmacological treatment are better detected by performing detailed analysis of the behavioural sequences. Further statistical studies should be carried out to improve the available methods to take into account complex experimental design.

(1) Haccou \& Meelis (1992) Statistical analysis of behavioural data, New York, Oxford University Press; (2) Bressers et al., (1995) Behav. Neurosci., 109:1158.

\section{TS11}

FUNCTIONAL INTEGRATION OF VISUAL, AUDITORY AND PROPRIOCEPTIVE SPATIAL MAPS.

F. Pavani* and E. Làdavas

Department of Psychology, University of Bologna, Italy

In the present study we investigated whether a derangement of the representation of visual space 
determined by a brain lesion can alter also the representation of acoustic space. To this aim we studied 12 right brain damaged patients with visual neglect, i.e. a derangement of the representation of visual space, in a free field sound localization task. To dissociate the contribution of the visuo-spatial deficit from the effects of the right hemisphere lesion, 12 right brain damaged patients without visual neglect and 7 healthy controls were also tested. Subjects were required to point manually to left, central and right sound sources, in a visual and in a blindfolded condition. The responding hand was located either in the left, central or right space. Results demonstrate a rightward error in sound localization responses of visual neglect patients in visual control condition, that was reduced when the visual input was eliminated, i.e. in blindfolded condition. Moreover, sound localization responses in left space were clearly biased in the direction of the starting position of the pointing hand. By contrast, the sound localization performance of right brain damaged patients without neglect was comparable to the performance of healthy subjects, in all experimental conditions. These findings are consistent with the view that the acoustic representation is modulated by the impaired visual representation and by the proprioceptive spatial map related to the position of the responding effector.In order to verify the relevance of the lateralized effectors in producing the systematic rightward errors in sound localization responses, the experiment was replicated by asking the patients to localize the acoustic stimulus by using the head, instead of the arm. In this experiment, the results showed a significant reduction of the localization errors towards right. The results will be discussed as an evidence of a functional interaction between different representations of the external space in the various sensory modalities.

\section{TS12}

\section{THE VISION OF THE HAND IN CODING PERIPERSONAL SPACE IN HUMANS}

A. Farnè ${ }^{l}$, G. Zeloni, ${ }^{2}$ G. di Pellegrino ${ }^{3}$, and E. Làdavas ${ }^{l}$

'Department of Psychology, University of Bologna, 40127 Italy and ${ }^{2}$ I.N.R.C.A. "I Fraticini", Firenze;

${ }^{3}$ Institute of Psychology, University of Urbino, Italy

Previous findings from our laboratory demonstrated the existence of a visual peripersonal space centred on the hand in humans and its modulatory effects on tactile perception. In brain damaged patients with tactile extinction, a strong modulatory effect of vision on touch perception was found when a visual stimulus was presented near the hand. In contrast, when the visual stimulus was presented far from the hand only a weak modulatory effect was found. The aim of the present study was to verify whether such cross-modal links between touch and vision in the peripersonal space centred on the hand could be mediated by proprioceptive signals specifying the current hand positions, or they directly reflect an interaction between two sensory modalities, i.e. vision and touch. To this aim, cross-modal effects were studied in two different experiments: one in which patients could see their hands and one in which the vision of their hands was prevented. The results showed strong modulatory effects of vision on touch perception when the visual stimulus was presented near the seen hand, and only mild effects when the vision of the hand was prevented. These findings are explained by referring to the activity of bimodal 
neurons in premotor and parietal cortex of macaque, which have tactile receptive fields on the hand, and corresponding visual receptive fields in the space immediately adjacent to the tactile fields. One important feature of these bimodal neurons is that their responsiveness to visual stimuli delivered near the body part is reduced or even extinguished when the view of the body part is prevented. This implies that, at least for the hand, the vision of the hand is crucial, with respect to the proprioceptive information, for determining the spatial mapping between vision and touch which takes place in the peripersonal space.

\title{
TS13
}

TRANSFER OF SPATIAL LEARNING FROM A VIRTUAL TO A REAL ENVIRONMENT, AND EFFECTS OF THE NATURE OF AVAILABLE INFORMATION

\author{
C. Thinus-Blanc*, L. Belingard, J.C. Malotaux, and P. Péruch \\ Center for Research in Cognitive Neuroscience, CNRS, Marseille, France
}

The present studies were aimed at examining 1) whether learning acquired from a virtual environment can be transferred to the corresponding real environment (Exp 1), and 2) to what extent the amount and/or quality of available information in the virtual situation facilitate(s) transfer to the actual situation (Exp2). In Exp 1, acquisition (by free exploration) took place in an actual environment (the campus of the CNRS) for one group («Real» group), and in a virtual version of the campus for the other («Virtual» group). Both groups were tested in both virtual and real situations (direction and distance estimates of locations). The Real group performed equally well on the real and on the virtual tests, while participants in the virtual group were more accurate when tests took place in the real situation then in the virtual one: The Real group performed better than the Virtual group on the virtual tests only. In Exp. 2, three virtual presentations of the CNRS campus were designed: One with only a subset of bare buildings (M1); another with the same buildings, and main road drawn so as to define a network of possible pathways (M2); the third one included buildings with details, lawns, hedges, trees, etc. and the network of main roads (M3). Participants from the three groups were then tested in the actual campus (direction and distance estimates of locations). Data show that the amount of details has no effect on the performance (no differences between M2 and M3). In contrast, the network of coloured pathways facilitates learning transfer (M2 and M3 better than M1). These data show that acquisition in a complex virtual environment is as accurate as when performed in the real situation. In addition, they suggest that the quality of information is more important than its amount. This opens the possibility to manipulate the available information so as to design virtual situations likely to facilitate further navigation in actual conditions. At a more theoretical level, these results address the question of the specific properties of environmental elements likely to become useful landmarks. 


\section{TS14}

\section{SPATIAL MEMORY IN ANXIOUS PATIENTS.}

I. Viaud-Delmon ${ }^{1,2}$ *, S. Lambrey ${ }^{2}$, R. Jouvent ${ }^{l}$, and A. Berthoz ${ }^{2}$

${ }^{l}$ CNRS UMR 7593, Hôpital de la Salpêtrière, ${ }^{2}$ LPPA, CNRS-Collège de France, 75005 Paris, France

Dysfunction of spatial orientation is already known to exist in agoraphobia. We addressed here the question of spatial orientation in anxious patients reporting experiencing dizziness. We investigated the memorization of a path previously navigated in a virtual environment. We studied in particular adaptation to a sensory discongruence during navigation and the transformation of sensory inputs (visual, vestibular, somato-sensory information and motor outflow) into a stored representation of the path. Subjects were equipped with a head-mounted display coupled with an electromagnetic sensor system. They were shown a virtual corridor along which they were (virtually) moving forward. The only instruction for them was to rotate themselves actively in order to change the direction of heading in the virtual corridor. We introduced a conflict between visual and idiothetic information by changing the gain between body rotation and visual scene. Three different gains were used. Each experiment contained two parts. I. Navigation: Subjects were asked to navigate twice in the same corridor. II. Memorized navigation: Subjects had to reproduce in darkness the previous navigation, by recalling their movement in the corridor from memory and rotating themselves appropriately. Additionally, the subjects were asked to draw their impression of the last corridor on a sheet of paper at the end of the experiment. Anxious patients managed to navigate accurately in the virtual corridor even in conflict conditions, but performed poorer than normal subjects in the memorized navigation. Spatial orientation deficits will be discussed in the general frame of sensory integration.

Supported by SmithKline Beecham

\section{TS15}

TEMPORAL INTEGRATION IN A SUBJECTIVE ACCENTUATION TASK: EXPERIMENTAL, CLINICAL AND DEVELOPMENTAL ASPECTS

E. Szelag ${ }^{l}$, J. Kowalska ${ }^{1}$, and E Pöppel ${ }^{2}$

${ }^{\prime}$ Nencki Institute of Exp. Biology, Warsaw, Poland and ${ }^{2}$ Institute for Medical Psychology, Munich, Germany

We report experimental evidence on temporal constraints of information processing in the domain of temporal integration (TI). With three experiments, using a subjective accentuation paradigm, we intended to answer the following questions: (1) is the left (speech) hemisphere is non-specifically involved in this TI? (2) are the classical language areas selectively associated with TI? (3) may the limits of TI alter during normal child development? We tested healthy volunteers (Exp.1), patients with unilateral focal brain lesions (Exp.2) and children aged 9-10 and 13-14 years (Exp.3). TI was assessed by measuring of the length of perceptual units consisting of a subjectively grouped number of metronome beats, presented 
either dichotically (Exp.1) or binaurally (Exp.2, 3). Usually, during listening to a string of identical stimuli, successive beats are bound together by subjectively accentuating every $\mathrm{x}$-th beat, resulting in a $\mathrm{TI}$ interval of less than $3 \mathrm{~s}$. The results indicated the lack of hemispheric asymmetry in the duration of TI (Exp.1). Specific TI disorders were found in Broca's aphasics which underlay probably language deficits characteristic for this aphasia syndrome (Exp.2). Moreover, the length of TI intervals depended on the frequency of presented beats (the higher frequency, the shorter TI). Although this relationship generally does not change during the normal cognitive development, the older children displayed longer integration than the younger ones (Exp.3). To conclude, the left hemisphere is not specifically involved in this TI process which is probably represented in the Broca's area. It is also postulated that the prefrontal cortex is a neuronal substrate for the developmental expansion of TI.

Supported by the KBN grant $N^{\circ} 4 . P O 5 E .09609$ and the EC grant $N^{\circ}$ CIPD CT 930126).

\section{TS16}

\section{MEMORY OF SELF-MOTION VELOCITY \& DURATION IN HUMAN}

I. Israël ${ }^{*}$, I. Siegler ${ }^{1}$, P. Dominey ${ }^{2}$, S. Rivaud-Péchoux ${ }^{3}$, B. Gaymard ${ }^{3}$, C. Pierrot-Deseilligny ${ }^{3}$, and A. Berthoz'

${ }^{1}$ LPPA-CNRS, Collège de France, Paris, ${ }^{2}$ ISC-CNRS, Bron, and ${ }^{3}$ Neurologie 1, INSERM-289, Hôpital de la Salpêtrière, France

The contribution of the vestibular system to the orientation of the body in space has long been suggested, and recent studies showed that the brain can estimate the traveled path solely from vestibular (and somatosensory) information. However, although the vestibulo-ocular reflex indicates head velocity sensitivity, subjective perception of velocity remains unknown. Moreover, when reproducing a previously imposed self-motion both motion amplitude and duration are reproduced. We then wondered whether time was reproduced as an effect of motion amplitude, or as independent information channel. We investigated the reproduction of plateau velocity and duration, when time is not supported by selfmotion perception since constant velocity is not sensed by the semi-circular canals. Subjects were seated on a motorized rotating chair turning about the vertical axis, in darkness. The chair was controlled by a PC microcomputer (stimulus), or the subjects could drive themselves the chair with a joystick (response). Stimulus rotations had a trapezoid velocity profile with 3 plateau velocities $(30,60$ and $90 \% \mathrm{~s})$ and 3 plateau durations $\left(2,3\right.$ and 4 s), leading to 9 angles (range $\left.82-496^{\circ}\right) .16$ healthy subjects $(8$ men and 8 women) had to reproduce the velocity and duration of the plateau of the imposed motion. PLATEAU VELOCITY (PV): PV gain was $0.97 \pm 0.19$. It was higher for the lowest stimulus velocity (SV, $30 \%$ : $1.16 \pm 0.19)$ and lower for the highest SV $(90 \%$ s: $0.86 \pm 0.09)$. It was correlated with stimulus plateau duration (PD), suggesting that a longer PD allowed reaching a higher PV.

PLATEAU DURATION (PD): PD gain was $0.77 \pm 0.30$. There was a negative correlation between PV gain and PD gain, as if subjects had retrieved plateau velocity and duration en-bloc. There was also a significant effect of stimulus PV upon PD gain ( $p<0.0003)$, PD gain being $0.70 \pm 0.27$ at $30 \%$ up to 

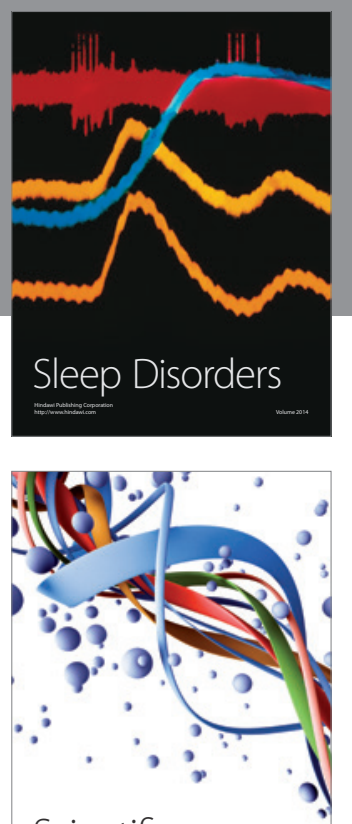

Scientifica
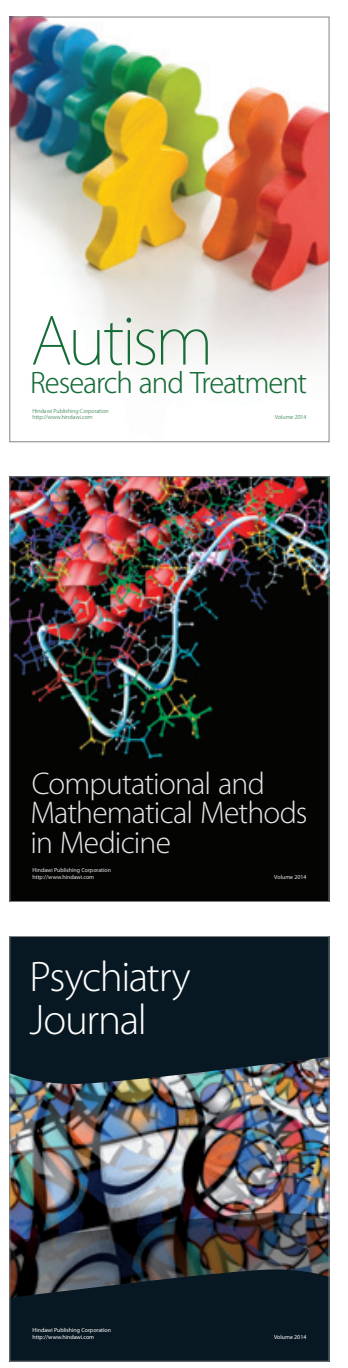
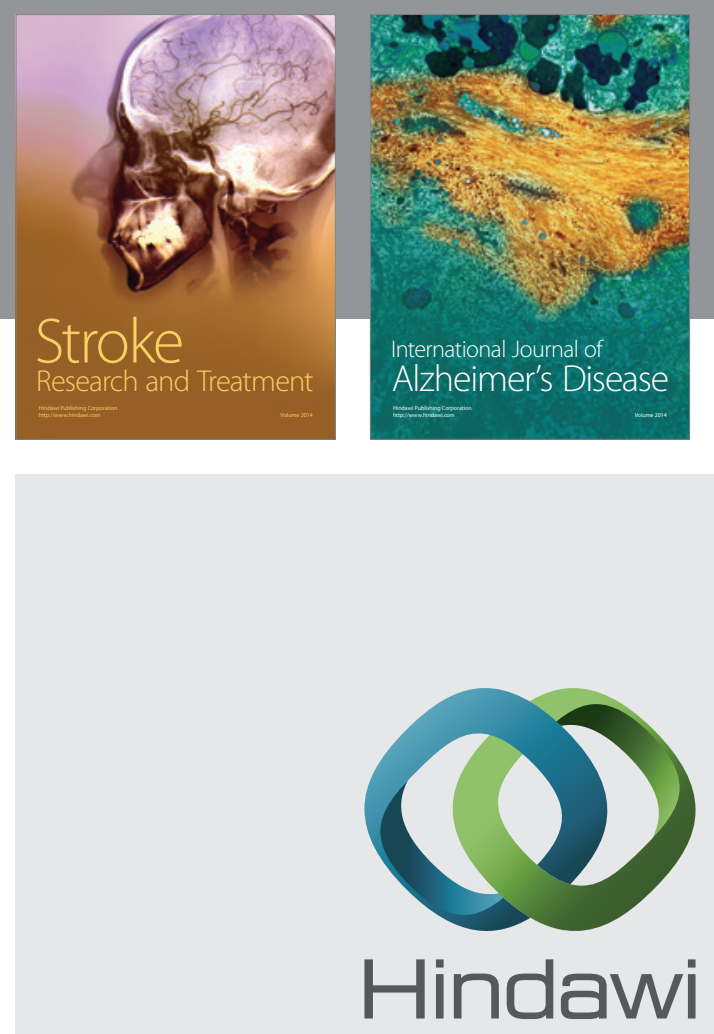

Submit your manuscripts at

http://www.hindawi.com
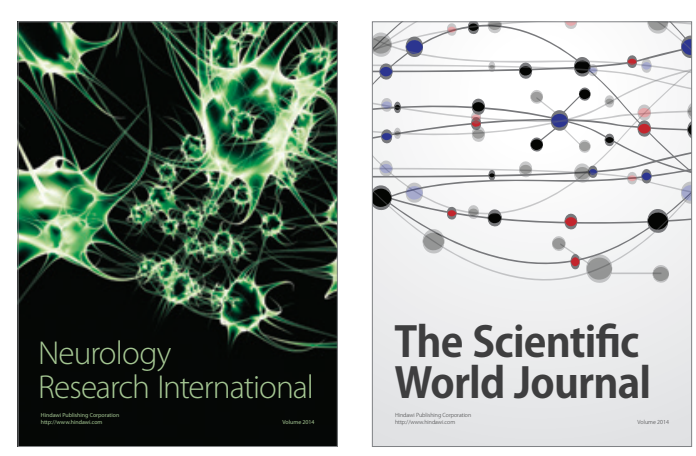

The Scientific World Journal

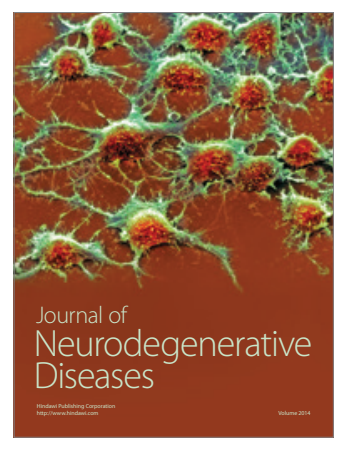

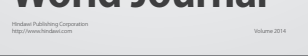

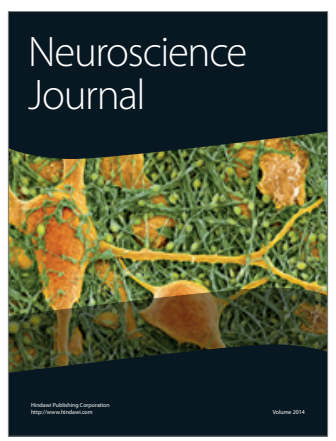

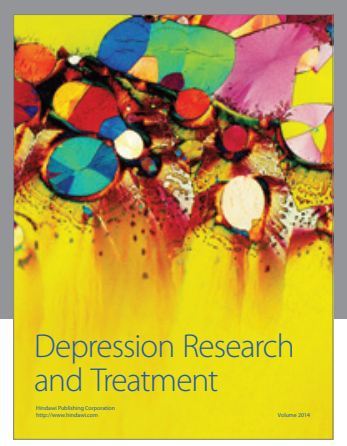
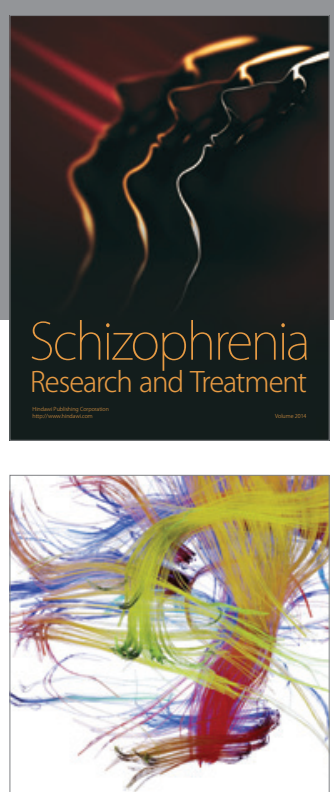

Brain Science

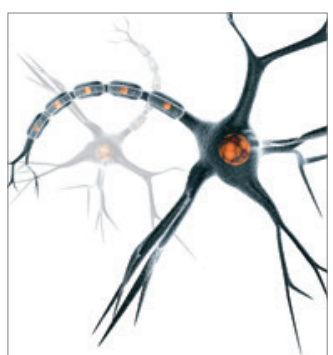

Neural Plasticity
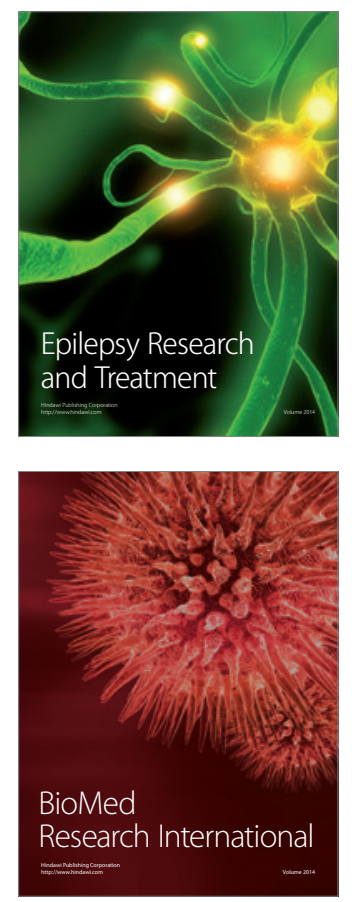

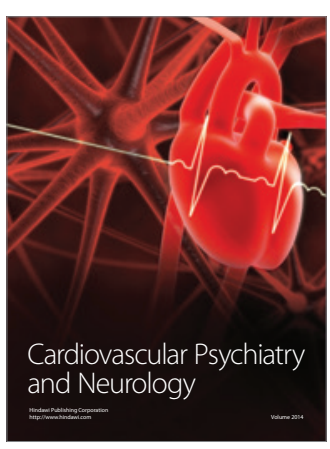

Parkinson's

Disease
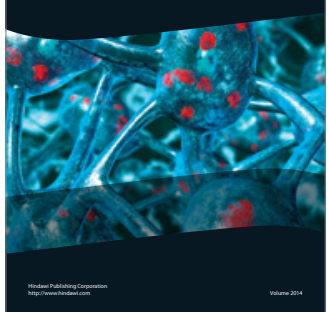